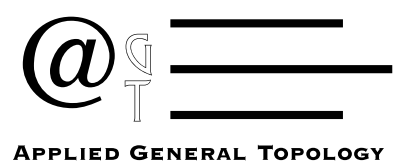

Applied General Topology

(C) Universidad Politécnica de Valencia

Volume 4, No. 2, 2003

pp. $263-279$

\title{
Locally convex approach spaces
}

\author{
M. Sioen AND S. Verwulgen
}

Dedicated to Professor S. Naimpally on the occasion of his $70^{\text {th }}$ birthday.

\begin{abstract}
We continue the investigation of suitable structures for quantified functional analysis, by looking at the notion of local convexity in the setting of approach vector spaces as introduced in [6]. We prove that the locally convex objects are exactly the ones generated (in the usual approach sense) by collections of seminorms. Furthermore, we construct a quantified version of the projective tensor product and show that the locally convex objects admitting a decent exponential law with respect to it are precisely the seminormed spaces.
\end{abstract}

2000 AMS Classification: 18B99, 18D15, 46A03, 46B04, 46M05, 46M15.

Keywords: approach vector space, topological vector space, locally convex space, locally convex approach space, Minkowski functional, Minkowski system, projective tensor product.

\section{INTRODUCTION AND PRELIMINARIES.}

In the setting of contemporary functional analysis the notion of a topological vector space plays a central role and over the last decennia, a great deal of effort has been done in studying their properties and relating them to different other fields in mathematics. However the totality of all topological vector spaces seems too large too handle at times to further develop and fine-tune this theory, as a lot of deep and interesting results (such as e.g Hahn-Banach type theorems, or suitable duality) only work well or are valid for certain subclasses of topological vector spaces. Certainly one of the most important better-behaved types of topological vector spaces are the locally convex ones, not in the least since they allow for the construction of several notions of tensor products. On the other hand, it is well-known from the classical theory that a topological vector space $X$ is locally convex if and only if it can be generated by a collection 
of seminorms, in the sense that there exists a set $\mathcal{P}$ of continuous seminorms on $X$ such that

$$
\{\{x \in X \mid p(x)<\varepsilon\} \mid p \in \mathcal{P}, \varepsilon>0\}
$$

is a base for the neighborhood system of 0 . It is also a well-known fact that this exactly means that $X$ as a topological space can be embedded in the (topological !) product of the family

$$
\left\{\left(X, \mathcal{T}_{\mu}\right) \mid \mu \in \mathcal{P}\right\}
$$

of semi-normable topological vector spaces (where $\mathcal{T}_{\mu}$ denotes the topology generated by $\mu$ ) in the following way:

$$
X \hookrightarrow \prod_{\mu \in \mathcal{P}}\left(X, \mathcal{T}_{\mu}\right): x \mapsto(\mu(x))_{\mu \in \mathcal{P}}
$$

Here another aspect, which is the central incentive for approach theory as introduced by $\mathrm{R}$. Lowen in $[8,9]$ appears: in (semi)normed spaces, canonical numerical information is intrinsically present in terms of the norm itself, but when we want to consider more general structures on vector spaces, essentially involving the construction of arbitrarily large set-indexed products, we classically automatically end up with topologies. So it seems that all the numerical information has been lost somewhere along the way: this also reflects in the fact that, for a given vector space $X$ and a given set $\mathcal{P}$ of seminorms on $X$, both $\mathcal{P}$ and $\left\{\alpha \mu \mid \alpha \in \mathbb{R}_{0}^{+}, \mu \in \mathcal{P}\right\}$ generate the same locally convex topology, although for each $\mu \in \mathcal{P}, \alpha \neq 1$, the seminorms $\mu$ and $\alpha \mu$ are essentially different from the numerical point of view! In the setting of topological vs. (pseudo-quasi-)metric spaces, a general solution to this problem was proposed by R. Lowen in $[8,9]$ through the introduction of the category Ap of approach spaces and contractions, in which arbitrary (set-indexed) products of metric objects can be formed yielding a canonical numerification of the product of the topologies underlying these objects. We refer to [8,9] for any further detailed information. On the other hand, R. Lowen together with the second named author introduced the notion of an approach vector space in [6] and it follows from the results proved there and also from [5] that the category ApVec of approach vector spaces and linear contractions is the right framework for quantified functional analysis, into which both the categories TopVec (of topological vector spaces and continuous maps) and pMetVec (of vector pseudometric vector spaces (in the sense of [16])(see also [6]) and linear non-expansive maps) are simultaneously embedded as full subcategories! Informally, one can say that ApVec solves the quantifying problem sketched above for $\mathbf{p M e t V e c}$ and TopVec, as Ap does for pMet and Top.

Locally convex topological spaces are not only prominent within the realm of topological vector spaces because they allow for stronger theorems, but also because some crucial constructions behave better under the assumption of local convexity.

One such construction, having paramount applications, as can be seen e.g. in [16], is the projective tensor product of locally convex topological spaces 
which reaches deeply into other subbranches such as e.g. the theory of nuclear operators [16] and vector valued integration [15].

The purpose of this paper is twofold: we want to put local convexity in the right perspective in the setting of approach vector spaces and then we want to use this locally convex approach structure (as we will call it) to create a canonical numerified counterpart of the classical topological projective tensor product, overlying the latter in a natural way. The main result of this paper is Theorem 3.9, where we exactly characterize the exponential behaviour of this quantified projective tensor product by showing that only seminormed spaces exhibit a categorically good notion of exponentiability.

First, let us mention that all vector spaces considered in this paper are vector spaces over the field of reals. We take [1] as our blanket reference for all facts of categorical nature and we refer the reader to $[8,9]$ for a detailed account on approach theory, together with the basic definitions, terminology and notations. Throughout the present paper, we will use the same terminology and notational conventions concerning approach vector spaces as defined in [6]. We briefly recall the most needed basic definitions and results, to make the text more self-contained.

Let $X$ be a vector space. For each $d \in[0,+\infty]^{X \times X}$, we define

$$
d[x]: X \rightarrow[0,+\infty]: y \mapsto d(x, y)
$$

and whenever $\mathcal{D} \subset[0,+\infty]^{X \times X}$, we let $\mathcal{D}[x]=\{d[x] \mid d \in \mathcal{D}\}$. Conversely, for $\phi: X \rightarrow[0,+\infty]$ we put

$$
\phi^{(2)}: X \times X \rightarrow[0,+\infty]:(x, y) \mapsto \phi(y-x)
$$

and whenever $\mathcal{C} \subset[0,+\infty]^{X}$, we put $\mathcal{C}^{(2)}=\left\{\phi^{(2)} \mid \phi \in \mathcal{C}\right\}$.

Throughout the whole framework of approach theory, the following concept of saturatedness, together with the obvious notion of saturation, for a collection of functionals plays a prominent role: we call $\mathcal{B} \subset \mathcal{C} \subset[0,+\infty]^{X}$ saturated in $\mathcal{C}$ if and only if the following condition holds:

$$
\forall \phi \in \mathcal{C}:(\forall \epsilon>0, \forall \omega<\infty \exists \psi \in \mathcal{B}: \phi \wedge \omega \leq \psi+\epsilon) \Rightarrow \phi \in \mathcal{B} .
$$

If $\mathcal{C}=[0, \infty]^{X}$, we simply talk about saturatedness. We write $\langle\mathcal{B}\rangle$ for the saturation of $\mathcal{B}$ within $[0, \infty]^{X}$, i.e.

$$
\langle\mathcal{B}\rangle:=\left\{\phi \in[0, \infty]^{X} \mid \forall \epsilon>0, \forall \omega<\infty \exists \psi \in \mathcal{B}: \phi \wedge \omega \leq \psi+\epsilon\right\},
$$

which obviously is the smallest saturated subset of $[0, \infty]^{X}$ containing $\mathcal{B}$.

Furthermore we call a functional $\phi \in[0,+\infty]^{X}$ sub-additive if

$$
\forall x, y \in X: \phi(x+y) \leq \phi(x)+\phi(y)
$$

and we say that $\phi$ is balanced if

$$
\forall x \in X, \forall \lambda \in[-1,1]: \phi(\lambda x) \leq \phi(x) .
$$

Finally $\phi$ is said to be absorbing if

$$
\forall x \in X, \forall \epsilon>0: \exists \delta>0: \forall \lambda \in[-\delta, \delta]: \phi(\lambda x) \leq \epsilon .
$$


A sub-additive, balanced and absorbing functional $\phi \in[0,+\infty]^{X}$ then is called a prenorm on $X$ and $\phi^{(2)}$ is called a vector pseudometric on $X$. (Also note that, as remarked in [6], prenorms only take finite values.)

Definition 1.1. [6] A pair $(X, \mathcal{A})$ consisting of a vector space $X$ and an approach system $\mathcal{A}=(\mathcal{A}(x))_{x \in X}$ is called an approach vector space if the following assertions hold

(AV1) For all $x \in X$ we have that

$$
\mathcal{A}(x)=\{y \mapsto \phi(y-x) \mid \phi \in \mathcal{A}(0)\} .
$$

(AV2) For all $\phi \in \mathcal{A}(0)$, for all $\epsilon>0$ and for all $\omega<\infty$, there exists $\psi \in \mathcal{A}(0)$ such that for all $x, y \in X$

$$
\phi(x+y) \wedge \omega \leq \psi(x)+\psi(y)+\epsilon .
$$

(AV3) For all $\phi \in \mathcal{A}(0)$, for all $\epsilon>0$ and for all $\omega<\infty$, there exists $\psi \in \mathcal{A}(0)$ such that for all $x \in X$ and for all $\lambda \in \mathbb{R}$ with $|\lambda| \leq 1$

$$
\phi(\lambda x) \wedge \omega \leq \psi(x)+\epsilon .
$$

(AV4) Every $\phi \in \mathcal{A}(0)$ is absorbing.

Note that $(\mathrm{AV} 1)$ together with $(\mathrm{AV} 2)$ exactly mean that $(X, \mathcal{A})$ is an approach group in the sense of [7]. A morphism between two approach vector spaces $\left(X, \mathcal{A}_{X}\right)$ and $\left(Y, \mathcal{A}_{Y}\right)$ is a linear map $f: X \rightarrow Y$ such that

$$
\forall \phi \in \mathcal{A}_{Y}(0): \phi \circ f \in \mathcal{A}_{X}(0),
$$

meaning that it is a contraction in the sense of [9]. Approach vector spaces and linear contractions then form a topological category over the category of vector spaces, as was shown in [6]. It was also proved in [6] that for a vector space $X$ and an approach system $\mathcal{A}=(\mathcal{A}(x))_{x \in X}$ the following assertions are equivalent

(1) $(X, \mathcal{A})$ is an approach vector space

(2) $\mathcal{A}(0)$ has a base of prenorms and

$$
\forall x \in X: \mathcal{A}(x)=\{y \mapsto \phi(y-x) \mid \phi \in \mathcal{A}(0)\} .
$$

If $\mathcal{G}$ is the gauge corresponding to $\mathcal{A}$ (see [9] for additional information) these assertions are moreover equivalent to saying that $\mathcal{G}$ has a base of vector pseudometrics.

\section{LOCALLY CONVEX APPROACH SPACES.} if

Note that, with $X$ a vector space, a functional $\phi \in[0, \infty]^{X}$ is called convex

$$
\forall x, y \in X, \forall \lambda \in[0,1]: \varphi(\lambda x+(1-\lambda) y) \leq \lambda \phi(x)+(1-\lambda) \phi(y),
$$

which obviously is equivalent to stating that, whenever we take a finite number of vectors $x_{1}, \ldots, x_{n}$ and real numbers $\lambda_{1}, \ldots, \lambda_{n} \in[0,1]$ with $\sum_{i=1}^{n} \lambda_{i}=1$, we have

$$
\phi\left(\sum_{i=1}^{n} \lambda_{i} x_{i}\right) \leq \sum_{i=1}^{n} \lambda_{i} \phi\left(x_{i}\right) .
$$


The following basic lemma will be the key tool for proving the main result (2.2) of this section: it provides a notion of a Minkowski-like functional associated with a given convex, balanced and absorbing functional, rather than with a given convex, balanced and absorbing set as suffices in the general topological vector space setting.

Lemma 2.1. Let $X$ be a vector space, $\phi \in[0, \infty]^{X}$ be a balanced, absorbing and convex functional and take $0<\omega<\infty$. Now define a new functional $\eta_{\varphi}^{\omega} \in[0, \infty]^{X}$ by

$$
\eta_{\phi}^{\omega}(x):=\inf \left\{\lambda>0 \mid \phi\left(\lambda^{-1} \omega x\right) \leq \omega\right\}, \quad x \in X .
$$

Then the following assertions hold:

(1) $\eta_{\phi}^{\omega}$ takes finite values and is a seminorm.

(2) $\eta_{\phi}^{\omega} \leq \phi$ on $\{\phi \geq \omega\}$.

(3) $\phi \leq \eta_{\phi}^{\omega}$ on $\{\phi \leq \omega\}$.

(4) $\eta_{\phi}^{\omega} \leq \phi \vee \omega \leq \phi+\omega$.

(5) $\phi \wedge \omega \leq \eta_{\phi}^{\omega}$.

Proof.

(1) The set $\{x \in X \mid \phi(\omega x) \leq \omega\}$ is absorbing, balanced and convex and $\eta_{\phi}^{\omega}$ is the Minkowski functional of this set.

(2) Take $x \in X$ such that $\phi(x) \geq \omega$. From the convexity of $\phi$ we have $\phi\left(\phi(x)^{-1} \omega x\right) \leq \phi(x)^{-1} \omega \phi(x)=\omega$. Thus $\phi(x) \in\left\{\lambda>0 \mid \phi\left(\lambda^{-1} \omega x\right) \leq\right.$ $\omega\}$ and so $\eta_{\phi}^{\omega}(x) \leq \phi(x)$.

(3) Let $x \in X$ such that $\phi(x) \leq \omega$. Suppose $\eta_{\phi}^{\omega}(x)<\phi(x)$.

Thus there exists $\lambda \in \mathbb{R}$ such that $0<\lambda<\phi(x)$ and $\phi\left(\lambda^{-1} \omega x\right) \leq \omega$. Because obviously also $\lambda \omega^{-1} \leq 1$, we would obtain that

$$
\begin{aligned}
\phi(x) & =\phi\left(\lambda \omega^{-1} \lambda^{-1} \omega x\right) \\
& \leq \lambda \omega^{-1} \phi\left(\lambda^{-1} \omega x\right) \\
& <\phi(x) \omega^{-1} \phi\left(\lambda^{-1} \omega x\right) \\
& \leq \phi(x),
\end{aligned}
$$

which is impossible.

(4) If $\phi(x) \geq \omega$ the inequality follows from 2 . above. If $\phi(x)<\omega$ note that $\phi(x)=\phi\left(\omega^{-1} \omega x\right) \leq \omega$ and hence $\eta_{\phi}^{\omega}(x) \leq \omega$.

(5) We only have to show the inequality for $\phi(x)>\omega$. Then for all $0<$ $\lambda \leq \omega$ we have that

$$
\omega<\phi\left(\frac{\lambda \omega}{\lambda \omega} x\right) \leq \phi\left(\lambda^{-1} \omega x\right)
$$

and hence $\eta_{\phi}^{\omega}(x) \geq \omega$.

As addressed in the introduction, it is a well-known fact that locally convex spaces (i.e. topological vector spaces which admit a base for the neighborhoods 
of 0 consisting of convex sets), can be generated by a so-called "gauge" of (continuous) seminorms. The following theorem is the quantified counterpart of it: it states that these approach vector spaces for which the local approach system (which is a lattice-theoretic ideal of functionals on $X$ !) at 0 is generated by convex functions (in the sense of saturation as defined above), can be generated in the same sense from seminorms. Compare this with [6] Theorem 5 (which was already quoted in the preliminaries), stating that any approach vector space has a generating set of prenorms.

Theorem 2.2. Let $(X, \mathcal{A})$ be an approach vector space. Then the following expressions are equivalent.

(1) $\mathcal{A}(0)$ has a base of seminorms, meaning there exists a set $\mathcal{P}$ of seminorms on $X$ which is an ideal base in the lattice $[0, \infty]^{X}$ and for which $\mathcal{A}(0)=\langle\mathcal{P}\rangle$,

(2) $\mathcal{A}(0)$ has a base of balanced, absorbing and convex functionals, meaning there exists a set $\mathcal{B}$ of absorbing and convex functionals on $X$ which is an ideal base in the lattice $[0, \infty]^{X}$ and for which $\mathcal{A}(0)=\langle\mathcal{B}\rangle$.

Proof.

$1 \Rightarrow 2$. This is clear, since any seminorm is a balanced, absorbing and convex function.

$2 \Rightarrow 1$. Let $\phi$ be a balanced, absorbing convex function in $\mathcal{A}(0)$ and fix $0<$ $\omega<\infty$. From Lemma 2.1 we deduce that $\eta_{\phi}^{\omega}$ is a seminorm and that $\phi \wedge \omega \leq \eta_{\phi}^{\omega}$. In order to show $\eta_{\phi}^{\omega} \in \mathcal{A}(0)$, pick $\epsilon>0$ and $\omega^{\prime}<\infty$. Let $m \in \mathcal{N}$ such that $\frac{\omega}{m} \leq \epsilon$. Since $(X, \mathcal{A})$ is an approach group $([6])$, there exists $\psi \in \mathcal{A}(0)$ such that for all $x_{1}, \ldots, x_{m} \in X$ :

$$
\phi\left(x_{1}+\cdots+x_{m}\right) \wedge m \omega^{\prime} \leq \psi\left(x_{1}\right)+\cdots+\psi\left(x_{m}\right) .
$$

From Lemma 2.1 we have $\eta_{\phi}^{\omega} \leq \phi+\omega$, hence for all $x \in X$ we have that

$$
\eta_{\phi}^{\omega}(m x) \wedge m \omega^{\prime} \leq \phi(m x) \wedge m \omega^{\prime}+\omega \leq m \psi(x)+\omega
$$

and therefore $\eta_{\phi}^{\omega} \wedge \omega^{\prime} \leq \psi+\epsilon$. Because $\mathcal{A}(0)$ has a base $\mathcal{B}$ of balanced, absorbing and convex functions, it follows that

$$
\mathcal{A}(0)=\left\langle\left\{\eta_{\varphi}^{\omega}|\varphi \in \mathcal{B}, 0<\omega<+\infty\rangle,\right.\right.
$$

and therefore automatically that

$$
\mathcal{A}(0)=\left\langle\left\{\bigvee_{i=1}^{n} \eta_{\varphi_{i}}^{\omega_{i}} \mid n \in \mathbb{N}_{0}, \varphi_{1}, \ldots, \varphi_{n} \in \mathcal{B}, 0<\omega_{1}, \ldots, \omega_{n}<+\infty\right\}\right\rangle,
$$

where the generating functionals in the last step still are seminorms.

This now justifies the following definition.

Definition 2.3. An approach vector space satisfying the properties of Theorem 2.2 is called a locally convex approach space. If we take locally convex approach spaces as objects and linear contractions (in the sense of $[8,9,6]$ as morphisms) we clearly obtain a full subcategory of ApVec, which we denote lcApVec. 
Whenever $X$ is a vector space, we call a set $\mathcal{M}$ of seminorms on $X$ a Minkowski system (on $X$ ) if it is a saturated ideal in the lattice of all seminorms on $X$.

If $(X, \mathcal{A})$ is a locally convex approach space, then

$$
\mathcal{M}_{\mathcal{A}}:=\{\eta \in \mathcal{A}(0) \mid \eta \text { is a seminorm }\}
$$

is a Minkowski system. Every Minkowski system is obtained in this way. Indeed, let $\mathcal{M}$ be a Minkowski system on $X$. Then we have, with

$$
\mathcal{A}_{\mathcal{M}}(x)=<\mathcal{M}^{(2)}[x]>,
$$

that $\left(X, \mathcal{A}_{\mathcal{M}}\right)$ is a locally convex approach space such that $\mathcal{M}=\mathcal{M}_{\mathcal{A}_{\mathcal{M}}}$ (since $\mathcal{M}$ is a saturated ideal of seminorms). Moreover by Theorem 2.2, the approach system $\mathcal{A}$ of a locally convex approach space is derived from a Minkowski system - in the sense of (2.1) because we have $\mathcal{A}=\mathcal{A}_{\mathcal{M}_{\mathcal{A}}}$. This even shows that, given a vector space $X$, there is a one-to-one correspondence between locally convex approach structures on $X$ and Minkowski systems on $X$. We will therefore also use notations like $(X, \mathcal{M})$ with $\mathcal{M}$ a Minkowski system on $X$ to denote lcApVec objects. Note that if $\mathcal{M}$ is a Minkowski system, then the collection $\left\{\eta^{(2)} \mid \eta \in \mathcal{M}\right\}$ is a gauge base for the gauge of the approach structure derived from $\mathcal{M}$.

Corollary 2.4. Let $\left(X_{1}, \mathcal{A}_{1}\right)$ and $\left(X_{2}, \mathcal{A}_{2}\right)$ be locally convex approach spaces with corresponding Minkowski systems $\mathcal{M}_{\mathcal{A}_{1}}$ and $\mathcal{M}_{\mathcal{A}_{2}}$ respectively. A linear map $f: X_{1} \rightarrow X_{2}$ is a morphism in lcApVec if and only if

$$
\forall \eta \in \mathcal{M}_{\mathcal{A}_{2}}: \eta \circ f \in \mathcal{M}_{\mathcal{A}_{1}} .
$$

To simplify the language, let us agree upon the following convention: in the sequel initial and final structures in (lc)ApVec (resp. (lc)TopVec) are understood to be taken with respect to the forgetful functor from (lc)ApVec (resp. (lc) TopVec) to Vec.

Theorem 2.5. lcApVec is initially closed in ApVec. Therefore $\mathbf{l c A p V e c}$ is topological over Vec.

Proof. Consider a class indexed source

$$
\left(f_{i}: X \rightarrow\left(X_{i}, \mathcal{A}_{i}\right)\right)_{i \in I}
$$

in lcApVec and let $\mathcal{A}$ be the initial ApVec-structure on $X$ for this source, viewed as a source in ApVec. For each $i \in I$, let $\mathcal{M}_{i}$ be the Minkowski system of $\left(X_{i}, \mathcal{A}_{i}\right)$. It was shown in [6] that the initial structures in ApVec are just the initial approach structures, and therefore it follows that

$$
\left\{\sup _{j=1}^{n} \eta_{i_{j}} \circ f_{i_{j}} \mid n \in \mathcal{N}_{0}, \forall j \in\{1, \ldots, n\}: i_{j} \in I, \eta_{i_{j}} \in \mathcal{M}_{i_{j}}\right\}
$$

is a base for $\mathcal{A}(0)$ which consists of seminorms, yielding that $(X, \mathcal{A})$ is a locally convex approach space. Hence $\left(f_{i}:(X, \mathcal{A}) \rightarrow\left(X_{i}, \mathcal{A}_{i}\right)\right)_{i \in I}$ is initial in lcApVec.

Corollary 2.6. The category $\mathbf{l c A p V e c}$ is concretely reflective in ApVec. 
Remark that if $(X, \mathcal{A})$ is an approach vector space then the set

$$
\{\eta \mid \eta \text { is a seminorm in } \mathcal{A}(0)\}
$$

can be taken as the Minkowski system of an lcApVec structure $\mathcal{A}^{\prime}$ on $X$. Clearly $\left(X, \mathcal{A}^{\prime}\right)$ is the reflection of $(X, \mathcal{A})$ in lcApVec.

Let $(X, \eta)$ be a seminormed space. Then $\eta^{(2)}$ is a pseudometric and $\left(X, \mathcal{A}_{\eta^{(2)}}\right)$ is a locally convex approach space. In the sequel we will make no distinction between a seminorm and the associated approach structure.

\section{Theorem 2.7.}

(1) A locally convex approach space which is at the same time a vector pseudometric space is a seminormed space.

(2) The category $\mathbf{s N o r m , ~ c o n s i s t i n g ~ o f ~ s e m i n o r m e d ~ s p a c e s ~ a n d ~ l i n e a r ~ n o n - ~}$ expansive maps between them, is embedded as a full subcategory of lcApVec. Moreover if $(X, \mathcal{A})$ is a locally convex approach space for which the approach structure is metric, then $(X, \mathcal{A})$ is a seminormed space.

Proof.

(1) Let $d$ be a vector pseudometric such that $\mathcal{A}_{d}(0)$ has a base of seminorms. Then the prenorm $d[0]$ is the supremum of seminorms and hence is a seminorm.

(2) It is easy to see that associating to every seminormed space $(X, \eta)$ the locally convex approach space $\left(X, \mathcal{A}_{\eta^{(2)}}\right)$ defines a concrete full embedding of sNorm into lcApVec.

To prove the second assertion, let $(X, \mathcal{A})$ be a locally convex approach space which at the same time is a metric object in Ap. This means that the approach gauge corresponding to $\mathcal{A}$ (see [9] for a definition) contains a largest metric $d$ which at the same time can be written as the pointwise supremum of a set of vector pseudometrics. Repeating the proof of [6] Theorem 16 now yields that $d$ itself is a vector pseudometric, hence $d[0]$ is a prenorm on $X$ for which $\mathcal{A}(0)=\langle\{d[0]\}\rangle$. Applying 1 now finishes the proof.

Theorem 2.8. sNorm is initially dense in lcApVec.

Proof. This is a consequence of Theorem 2.2: if $(X, \mathcal{A})$ is a locally convex approach space, then the source

$$
\left(\operatorname{id}_{X}:(X, \mathcal{A}) \rightarrow(X, \eta)\right)_{\eta \in \mathcal{M}_{\mathcal{A}}}
$$

is initial in lcApVec.

\section{Theorem 2.9.}

(1) lcTopVec is embedded as a full subcategory of $\mathbf{l c A p V e c}$ 
(2) lcTopVec is concretely coreflective in $\mathbf{l c A p V e c . ~ M o r e o v e r ~ w e ~ h a v e ~}$ the commutation of

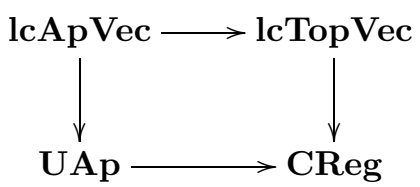

where the horizontal arrows are the concrete coreflectors and the vertical arrows the forgetful functors.

(3) If $(X, \mathcal{A})$ is a locally convex approach space such that the underlying approach system is topological then $(X, \mathcal{A})$ is a locally convex topological space.

Proof. (1) A locally convex topological space is also an approach vector space $([6])$ and since the characteristic function of a convex set is a convex function we know the approach system of 0 has a base of convex functions.

(2) Let $(X, \mathcal{A})$ be a locally convex approach space and let $\mathcal{T}$ be the topological coreflection of $(X, \mathcal{A})$ in Ap. From [6], Theorem 21, we know that $(X, \mathcal{T})$ is a topological vector space. Since $\mathcal{M}_{\mathcal{A}}$ is a base for $\mathcal{A}(0)$, we know that

$$
\{\{n \leq \epsilon\} \mid \epsilon>0, n \in \mathcal{M}\}
$$

is a base of convex sets for the neighborhood system of 0 .

(3) Let $(X, \mathcal{A})$ be a topological locally convex approach space. Then by 2. above, $(X, \mathcal{A})$ equals its lcTopVec coreflection and is thus a locally convex topological space.

Proposition 2.10. Let $\mathcal{M}$ be an ideal of seminorms. Then $\mathcal{M}$ is the Minkowski system of a locally convex topological space if and only if

$$
\forall \lambda \in \mathbb{R}^{+}, \forall \eta \in \mathcal{M}: \lambda \eta \in \mathcal{M} .
$$

Proof. The Minkowski system of a locally convex topological space is just the set of Minkowski functionals of the balanced, convex and absorbing open sets and hence it satisfies (2.2). Conversely $\mathcal{M}$ is saturated if it satisfies (2.2). Moreover, in this case the associated approach system is topological and the result follows from Theorem 2.9 .

Corollary 2.11. lcTopVec is initially closed in lcApVec.

Corollary 2.12. lcTopVec is initially closed in TopVec ([3]) and hence lcTopVec is concretely reflective in TopVec.

Corollary 2.13. lcTopVec is finally closed in lcApVec

Corollary 2.14. Let $(X, \mathcal{M})$ be a locally convex approach space. Then the set

$$
\left\{\lambda \eta \mid \lambda \in \mathbb{R}^{+}, \eta \in \mathcal{M}\right\}
$$

is the Minkowski system of the topological coreflection of $(X, \mathcal{M})$. 
In order to sketch the general results we extend the summarizing diagram of $[6]$ to the following commutative diagram:

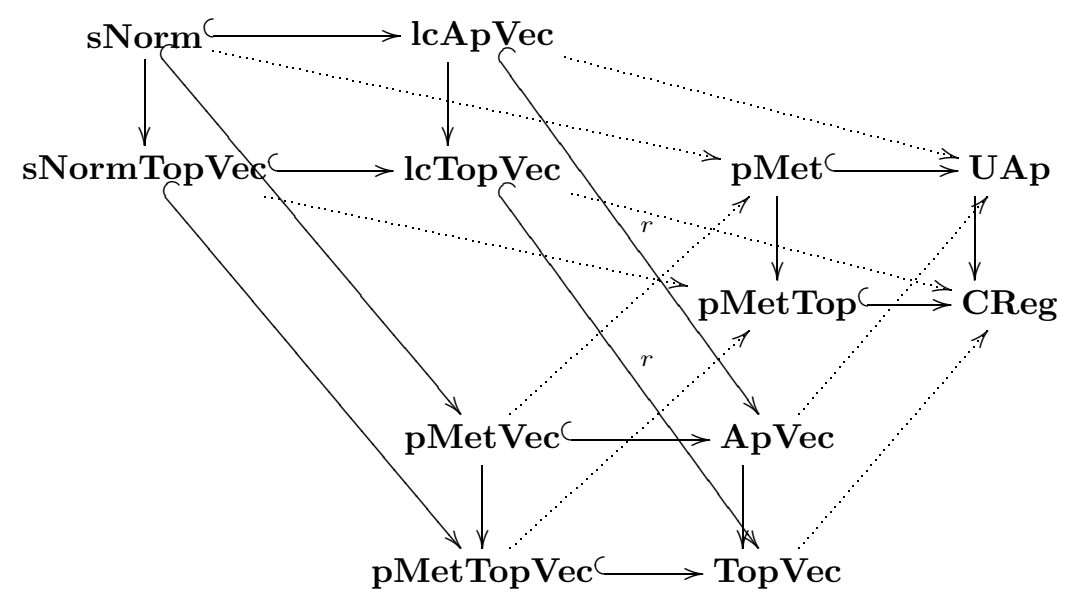

Here the categories in the top layer are the approach versions of those in the bottom layer. The horizontal embeddings are initially dense and the dashed and vertical arrows are the usual forgetful functors.

Since lcTopVec is not finally closed in TopVec ([3]) and the latter category is finally closed in ApVec ([6], corollary 22), by Theorem 2.9 we have lcApVec is not finally closed in ApVec.

Note that normable topological vector spaces are not the result of imposing the convexity condition on the metrizable topological vector spaces; there's a subtle difference with Theorem 2.7: see [14] for a locally convex topological vector space for which the topology is metrizable but not normable.

\section{The PROJECTIVE TENSOR PRODUCT AND BICONTRACTIONS.}

In the sequel $X, Y$ and $Z$ are presumed to be locally convex approach vector spaces with approach and Minkowski systems $\mathcal{A}_{X}, \mathcal{A}_{Y}, \mathcal{A}_{Z}$ and $\mathcal{M}_{X}, \mathcal{M}_{Y}$, $\mathcal{M}_{Z}$ respectively.

Every locally convex topology being defined by a family of seminorms, in [4] the projective tensor product of two locally convex topological vector spaces is introduced using these seminorms. Recall from [16] that, if $\mu$ and $\nu$ are seminorms on respectively $X$ and $Y$, the map

$$
\mu \otimes \nu: X \otimes Y \rightarrow \mathbb{R}^{+}: u \mapsto \mu \otimes \nu(u):=\inf \left\{\sum_{i=1}^{n} \mu\left(x_{i}\right) \nu\left(y_{i}\right) \mid \sum_{i=1}^{n} x_{i} \otimes y_{i}=u\right\}
$$

is a seminorm on the algebraic tensor product $X \otimes Y$ of the vector spaces $X$ and $Y$ which, moreover, satisfies the following identity:

$$
\forall x \in X, \forall y \in Y: \mu \otimes \nu(x \otimes y)=\mu(x) \nu(y) .
$$

We now in the obvious way can define a quantified version of the projective tensor product in the realm of locally convex approach spaces. 
Definition 3.1. The projective tensor product $X \otimes Y$ is the algebraic tensor product of $X$ and $Y$ endowed with $\mathcal{M}_{X \otimes Y}$, the Minkowski system generated by the set $\left\{\mu \otimes \nu \mid \mu \in \mathcal{M}_{X}, \nu \in \mathcal{M}_{Y}\right\}$.

Note that $\left\{\mu \otimes \nu \mid \mu \in \mathcal{M}_{X}, \nu \in \mathcal{M}_{Y}\right\}$ indeed is a base for an ideal in the set of all seminorms on $X \otimes Y$, because for all $\mu, \mu^{\prime} \in \mathcal{M}_{X}, \nu, \nu^{\prime} \in \mathcal{M}_{Y}$ clearly

$$
(\mu \otimes \nu) \vee\left(\mu^{\prime} \otimes \nu^{\prime}\right) \leq\left(\mu \vee \mu^{\prime}\right) \otimes\left(\nu \vee \nu^{\prime}\right)
$$

and $\mathcal{M}_{X}, \mathcal{M}_{Y}$ are closed for taking finite suprema.

Theorem 3.2. The locally convex topological coreflection of $\left(X \otimes Y, \mathcal{M}_{X \otimes Y}\right)$ is the projective tensor product of the locally convex topological coreflections of $X$ and $Y$.

Proof. This follows from [16].

Corollary 3.3. The projective tensor product of topological locally convex approach spaces is again topological. Moreover if we restrict the projective tensor product to lcTopVec we obtain the classical projective tensor product.

The following very useful lemma, tells us that the saturation condition we have used throughout the whole paper for the approach system $\mathcal{A}(0)$ within $[0, \infty]^{X}$, corresponds to a more elegant and especially more manageable form when restricted to the case of the associated Minkowski system $\mathcal{M}$ within the set of all seminorms on the vector space $X$.

Lemma 3.4. Let $\mathcal{M}$ be a Minkowski system on a vector space $W$. For a seminorm $\eta$ on $W$, the following equivalence holds:

$$
\eta \in \mathcal{M} \Leftrightarrow \forall \epsilon>0: \exists \mu \in \mathcal{M}: \eta \leq \mu(1+\epsilon) .
$$

Proof. Suppose $\eta \in \mathcal{M}$. Let $\epsilon>0$. Since $\mathcal{M}$ is saturated there exists $\mu \in \mathcal{M}$ such that $\eta \wedge 1 \leq \mu+\epsilon^{\prime}$, where $\epsilon^{\prime}=1-\frac{1}{1+\epsilon}$. Then for all $x$ in $W$ we have (with $n(x) \neq 0) \eta\left(\frac{x}{\eta(x)}\right) \leq \mu\left(\frac{x}{\eta(x)}\right)+\epsilon^{\prime}$. Therefore $\eta(x)\left(1-\epsilon^{\prime}\right) \leq \mu(x)$, from which $\eta(x) \leq \frac{\mu(x)}{1-\epsilon^{\prime}}=\mu(x)(1+\epsilon)$ follows, for all $x$ in $W$.

Conversely let $\epsilon>0$, let $0<\omega<\infty$ and let $\mu$ in $\mathcal{M}$ such that $\eta \leq\left(1+\epsilon^{\prime}\right) \mu$, where $0<\epsilon^{\prime}<\min \left\{1, \frac{\epsilon}{\omega}\right\}$ is fixed. Note that $1 \geq 1-\epsilon^{\prime 2}=\left(1-\epsilon^{\prime}\right)\left(1+\epsilon^{\prime}\right)>0$. Hence $\eta \leq \mu\left(1+\epsilon^{\prime}\right) \leq \frac{\mu}{1-\epsilon^{\prime}}$. Therefore $\eta \leq \mu+\epsilon^{\prime} \eta$. Now take $x \in W$. If $\eta(x) \leq \omega$, that is $\eta\left(\frac{x}{\omega}\right) \leq 1$, then $\eta\left(\frac{x}{\omega}\right) \leq \mu\left(\frac{x}{\omega}\right)+\epsilon^{\prime} \eta\left(\frac{x}{\omega}\right) \leq \mu\left(\frac{x}{\omega}\right)+\epsilon^{\prime}$. Thus $\eta(x) \leq \mu(x)+\epsilon^{\prime} \omega \leq \mu(x)+\epsilon$. Now suppose that $\eta(x)>\omega$ and at the same time that $\mu(x)+\epsilon<\omega$. Then $1>\mu\left(\frac{x}{\omega}\right)+\frac{\epsilon}{\omega}>\mu\left(\frac{x}{\omega}\right)+\epsilon^{\prime}$. Since this implies that $\mu\left(\frac{x}{\omega}\right) \leq 1$ we also obtain $1>\mu\left(\frac{x}{\omega}\right)\left(1+\epsilon^{\prime}\right) \leq \eta\left(\frac{x}{\omega}\right)$, which is impossible. We thus have proved $\eta \wedge \omega \leq \mu+\epsilon$.

Let $\left(\mu_{i}\right)_{i \in I}$ be a collection of seminorms on a vector space $X$. Note that the infimum $\operatorname{sinf}_{i \in I} \mu_{i}$ in the set of all seminorms on $X$ exists.

Definition 3.5. A binorm $b$ on the vector space $X \times Y$ is a function

$$
b: X \times Y \rightarrow \mathbb{R}^{+}
$$

such that, for all $\lambda \in \mathbb{R}$, for all $x, x^{\prime} \in X$ and $y, y^{\prime} \in Y$ : 
(1) $b(\lambda x, y)=|\lambda| b(x, y)=b(x, \lambda y)$

(2) $b\left(x+x^{\prime}, y\right) \leq b(x, y)+b\left(x^{\prime}, y\right)$

(3) $b\left(x, y+y^{\prime}\right) \leq b(x, y)+b\left(x, y^{\prime}\right)$.

Let $\left(b_{i}\right)_{i \in I}$ be a collection of binorms on $X \times Y$. Then the infimum $\operatorname{binf}_{i \in I} b_{i}$ exists in the set of all binorms on $X \times Y$. Moreover we have, for $\left(\mu_{i}\right)_{i \in I}$ and $\left(\nu_{j}\right)_{j \in J}$ a collection of seminorms on $X$ respectively $Y$, that

$$
\left(\left(\mu_{i} \circ \operatorname{pr}_{X}\right)\left(\nu_{j} \circ \operatorname{pr}_{Y}\right)\right)_{(i, j) \in I \times J}
$$

is a collection of binorms on $X \times Y$ and

$\left(\left(\operatorname{sinf}_{i \in I} \mu_{i}\right) \circ\right.$ textrmpr $\left.r_{X}\right)\left(\left(\operatorname{sinf}_{j \in J} \nu_{j}\right) \circ \operatorname{pr}_{Y}\right)=\operatorname{binf}_{(i, j) \in I \times J}\left(\mu_{i} \circ \operatorname{pr}_{X}\right)\left(\nu_{j} \circ \operatorname{pr}_{Y}\right)$.

If $f: X \times Y \rightarrow Z$ is a bilinear map, we write

$$
\bar{f}: X \otimes Y \rightarrow Z
$$

for its obvious, linear factorization over the tensor product $X \otimes Y$, which is well-defined by putting $\bar{f}(x \otimes y)=f(x, y)$ for all $x \in X$ and $y \in Y$.

Proposition 3.6. Let $f: X \times Y \rightarrow Z$ be a bilinear map. The following are equivalent.

(1) The linear lift $\bar{f}:\left(X \otimes Y, \mathcal{M}_{X \otimes Y}\right) \rightarrow\left(Z, \mathcal{M}_{Z}\right)$ is a contraction.

(2) $\forall \eta \in \mathcal{M}_{Z}, \forall \omega<\infty, \forall \epsilon>0: \exists \mu \in \mathcal{M}_{X}: \exists \nu \in \mathcal{M}_{Y}: \forall x \in X, \forall y \in Y:$

$$
\eta(f(x, y)) \wedge \omega \leq \mu(x) \nu(y)+\epsilon .
$$

(3) $\forall \eta \in \mathcal{M}_{Z}, \forall \epsilon>0: \exists \mu \in \mathcal{M}_{X}: \exists \nu \in \mathcal{M}_{Y}: \forall x \in X, \forall y \in Y:$

$$
\eta(f(x, y)) \leq \mu(x) \nu(y)(1+\epsilon) .
$$

(4) $\forall \eta \in \mathcal{M}_{Z}: \exists \mu \in \mathcal{M}_{X}: \exists \nu \in \mathcal{M}_{Y}: \forall x \in X, \forall y \in Y:$

$$
\eta(f(x, y)) \leq \mu(x) \nu(y) .
$$

Proof. $\quad 1 \Leftrightarrow 2 \Leftrightarrow 3$. Straightforward.

$3 \Rightarrow 4$. Take $\eta \in \mathcal{M}_{Z}$ and choose, for all $n \in \mathcal{N}_{0}, \mu_{n}^{\prime} \in \mathcal{M}_{X}$ and $\nu_{n}^{\prime} \in \mathcal{M}_{Y}$ such that for all $x \in X$ and $y \in Y \eta(f(x, y)) \leq$ $\mu_{n}^{\prime}(x) \nu_{n}^{\prime}(y)(1+1 / n)$. Let $\mu_{n}=\sup _{k \leq n} \mu_{k}^{\prime}$ and let $\nu_{n}=\sup _{k \leq n} \nu_{k}^{\prime}$. Define $\mu=\operatorname{sinf}_{n \in \mathcal{N}_{0}} \mu_{n}(1+1 / n)$ and $\nu=\operatorname{sinf}_{n \in \mathcal{N}_{0}} \nu_{n}(1+1 / n)$. Then $\mu$ is in $\mathcal{M}_{X}$ and $\mu$ is in $\mathcal{M}_{Y}$ and we have

$$
\begin{aligned}
& \left(\mu \circ \operatorname{pr}_{X}\right)\left(\nu \circ \operatorname{pr}_{Y}\right) \\
= & \left(\left(\operatorname{sinf}_{m \in \mathcal{N}_{0}} \mu_{m}(1+1 / m)\right) \circ \operatorname{pr}_{X}\right)\left(\left(\operatorname{sinf}_{n \in \mathcal{N}_{0}} \nu_{n}(1+1 / n)\right) \circ \operatorname{pr}_{Y}\right) \\
= & \operatorname{binf}_{(n, m) \in \mathcal{N}_{0}^{2}}\left(\mu_{m} \circ \operatorname{pr}_{X}\right)\left(\nu_{n} \circ \operatorname{pr}_{Y}\right)(1+1 / m)(1+1 / n) \\
\geq & \operatorname{binf}_{(n, m) \in \mathcal{N}_{0}^{2}}\left(\mu_{\min (m, n)} \circ \operatorname{pr}_{X}\right)\left(\nu_{\min (m, n)} \circ \operatorname{pr}_{Y}\right)(1+1 / \min (m, n)) \\
= & \operatorname{binf}_{n \in \mathcal{N}_{0}}\left(\mu_{n} \circ \operatorname{pr}_{X}\right)\left(\nu_{n} \circ \operatorname{pr}_{Y}\right)(1+1 / n) \\
\geq & \operatorname{binf}_{n \in \mathcal{N}_{0}} \eta \circ f \\
= & \eta \circ f .
\end{aligned}
$$

The implication $4 \Rightarrow 1$ is obvious. 
Definition 3.7. If $f$ satisfies (one of) the above properties it is called a bicontraction.

\section{Example 3.8.}

(1) Consider $\mathbb{R}$, equipped with $|\cdot|$. Then scalar multiplication $M: \mathbb{R} \times X \rightarrow$ $X$ is a bicontraction. Moreover $\bar{M}: \mathbb{R} \otimes X \rightarrow X$ is an isomorphism between locally convex approach spaces.

(2) The map $t: X \times Y \rightarrow X \otimes Y:(x, y) \mapsto x \otimes y$ is a bicontraction. Hence for all $X$ we have a functor $-\otimes X: \mathbf{l c A p V e c} \rightarrow \mathbf{l c A p V e c}$.

Note that it is clear from the definition that for seminormed spaces $(X, \eta)$ and $(Y, \mu)$, their projective tensor product in $\mathbf{l c A p V e c}$ corresponds exactly to their "classical" seminormed tensor product $(X \otimes Y, \eta \otimes \mu)$, so that no notational ambiguity can arise.

From the thoroughly studied tensor product in sNorm (see e.g. [15]) we know however that there is much more to say in this case: the projective tensor product defines a symmetric bifunctor

$$
-\otimes-: \text { sNorm } \times \text { sNorm } \rightarrow \text { sNorm }
$$

which determines a so-called symmetric monoidal closed structure. This means that for every seminormed space $X=(X, \eta)$ the functor

$$
-\otimes X: \mathbf{s N o r m} \rightarrow \mathbf{s N o r m}
$$

has a right adjoint, a so-called inner hom functor

$$
(-)^{X}: \mathbf{s N o r m} \rightarrow \text { sNorm, }
$$

which is completely determined by putting for every seminormed space $Y=$ $(Y, \mu) Y^{X}$ to be the vector space of all linear continuous functions from $X$ to $Y$, equipped with the seminorm $\mu_{\eta}$, given by

$$
\mu_{\eta}(f)=\sup _{\eta(x) \leq 1} \mu(f(x)) .
$$

Alternatively, this means that for all $X, Y, Z \in|\mathbf{s N o r m}|$

$$
Z^{X \otimes Y} \simeq\left(Z^{Y}\right)^{X}
$$

where the isomorphism is natural in $X, Y, Z$.

For the locally convex topological case, the projective tensor product in lcTopVec (to which our quantified version reduces according to Theorem 3.2) determines a symmetric bifunctor

$$
-\otimes-: \text { lcTopVec } \times \text { lcTopVec } \rightarrow \text { lcTopVec }
$$

which however no longer gives rise to a symmetric monoidal closed structure! (Note that as can be seen e.g. from [17] other interesting symmetric monoidal closed structures on categories of barreled-like locally convex topological spaces exist.)

Also in the case of locally convex approach spaces, the projective tensorproduct we introduced gives rise to a bifunctor

$$
-\otimes-: \text { lcApVec } \times \text { lcApVec } \rightarrow \text { lcApVec. }
$$


Our main theorem of this section exactly shows how far this bifunctor is away from determining a symmetric monoidal closed structure on lcApVec, by classifying those locally convex approach spaces $X$ for which $-\otimes X$ has a right adjoint.

We recall the nice exponential law (3.3) as a simple corollary to this theorem.

Theorem 3.9. The functor $-\otimes X$ has a right adjoint if and only if $X$ is a seminormed space.

Proof. To begin with, suppose $-\otimes X$ has a right adjoint. We now proceed in several steps.

(1) Fix $Y$ and let $W$ be a locally convex approach space and $\epsilon: W \otimes X \rightarrow Y$ be a linear contraction such that for all $Z$ and for all $f: Z \otimes X \rightarrow Y$ linear contraction there exists a unique linear contraction $\hat{f}: Z \rightarrow W$ such that the following diagram is commutative:

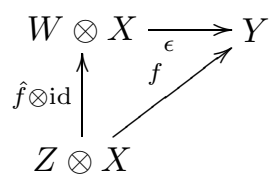

Define $\sigma: W \rightarrow L(X, Y)$ by $\sigma(w)(x)=\epsilon(w \otimes x)$, where $L(X, Y)$ is the set of all linear maps from $X$ to $Y$. Let $Y^{X}$ be the subspace $\sigma(W)$ of $L(X, Y)$. Note that, for all $\nu \in \mathcal{M}_{W}, \sigma(\nu)$, defined by $\sigma(\nu)(a)=$ $\inf _{\sigma(w)=a} \nu(w)$, is a seminorm on $Y^{X}$. Let $M_{Y^{X}}$ be the Minkowski system generated by $\left\{\sigma(\nu) \mid \nu \in \mathcal{M}_{W}\right\}$. Then $\sigma$ is a linear contraction. For further reference, $\mathcal{M}_{Y^{X}}$ is called the function space structure.

(2) We claim that

$$
e v: Y^{X} \times X \rightarrow Y:(a, x) \mapsto a(x)
$$

is a bicontraction. To prove this let $\eta \in \mathcal{M}_{Y}$ and let $\epsilon>0$. Let $\mu \in \mathcal{M}_{X}$ and $\nu \in \mathcal{M}_{W}$ such that for all $w$ in $W$ and $x$ in $X$ we have $\eta(\epsilon(w \otimes x)) \leq \nu(w) \mu(x)(1+\epsilon)$. Fix $w$ in $W$. Let $w^{\prime}$ in $\sigma^{-1}(\sigma(w))$. Then $\eta(e v(\sigma(w), x))=\eta\left(e v\left(\left(\sigma\left(w^{\prime}\right), x\right)\right)=\eta\left(\epsilon\left(w^{\prime} \otimes x\right)\right) \leq \nu\left(w^{\prime}\right) \mu(x)(1+\epsilon)\right.$. Hence $\eta(e v(\sigma(w), x)) \leq \inf _{w^{\prime} \in \sigma^{-1}(\sigma(w))} \nu\left(w^{\prime}\right) \mu(x)(1+\epsilon)=\sigma(\nu)(\sigma(w))$ $\mu(x)(1+\epsilon)$.

(3) Note that the triangle

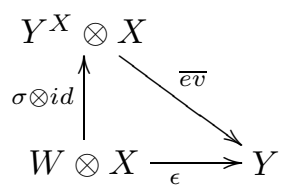

is commutative. Take $f: Z \otimes X \rightarrow Y$ a linear contraction, let $\hat{f}: Z \rightarrow$ $W$ be such that $f=\epsilon \circ(\hat{f} \otimes i d)$. Then we have following commutative 
diagram

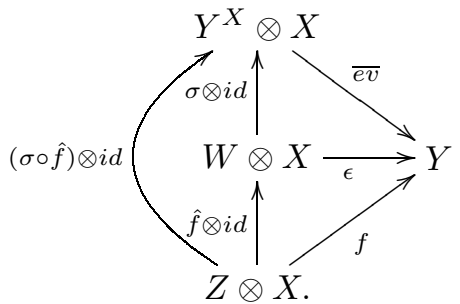

So we have $f=\overline{e v} \circ((\sigma \circ \hat{f}) \otimes i d)$. Let $g: Z \rightarrow Y^{X}$ be a linear contraction such that $f=\overline{e v} \circ g \otimes i d$. Then for all $x$ in $X$ and $z$ in $Z$ we have

$$
\begin{aligned}
g(z)(x) & =e v \circ(g \times i d)(z, x) \\
& =\overline{e v} \circ(g \otimes i d)(z \otimes x) \\
& =f(z \otimes x) \\
& =\epsilon(\hat{f}(z) \otimes x) \\
& =\sigma(\hat{f}(z))(x) \\
& =(\sigma \circ \hat{f})(z)(x) .
\end{aligned}
$$

This means $g=\sigma \circ \hat{f}$.

(*) For all linear contractions $f: Z \otimes X \rightarrow Y$, there exists a unique linear contraction $\tilde{f}: Z \rightarrow Y^{X}$ such that $f=\overline{e v} \circ(\tilde{f} \otimes i d)$.

(4) Since $e v: Y^{X} \times X \rightarrow Y$ is a bicontraction, we know that $Y^{X}$ contains only continuous linear maps from $X$ to $Y$. Let $a: X \rightarrow Y$ be a linear continuous map. Then the map $a^{\prime}: \mathbb{R}_{\mathcal{T}} \times X \rightarrow Y:(\lambda, x) \mapsto \lambda a(x)$ is a linear contraction, where $\mathbb{R}_{\mathcal{T}}$ is endowed with the Euclidean topology. Then we have that $\tilde{a}^{\prime}(1)=a$. This shows that $Y^{X}$ is the set of all continuous linear maps.

(5) In the above, let $Y=\mathbb{R}$ equipped with the absolute value. We write $X^{\prime}$ for the space of linear continuous maps from $X$ to $\mathbb{R}$, let $\mathcal{M}_{X^{\prime}}$ be the function space structure. Define, for all $\eta$ in $\mathcal{M}_{X}$ and $a$ in $X^{\prime}$,

$$
|a|_{\eta}=\sup _{\eta(x) \leq 1}|a(x)| .
$$

So we have a map $|\cdot|_{\eta}: X^{\prime} \rightarrow[0, \infty]$. Take $\xi \in \mathcal{M}_{\mathbb{R}^{X}}$ and $\eta \in \mathcal{M}_{X}$ such that, for all $a \in X^{\prime}$ and $x \in X$ we have $|a(x)| \leq \xi(a) \eta(x)$. Then it follows

$$
|\cdot|_{\eta} \leq \xi
$$

Take $\mu \in \mathcal{M}_{X}$. Note that $\mu \vee \eta \geq \eta$, hence

$$
|\cdot|_{\mu \vee \eta} \leq|\cdot|_{\eta}
$$

So, from (3.4), we see $|\cdot|_{\eta}$ is a seminorm on $X$. Note also that $|a(x)| \leq$ $|a|_{\mu \vee \eta}(\mu \vee \eta(x))$. So if we consider $X^{\prime}$ equipped with $|\cdot|_{\eta \vee \mu}$ then $e v: X^{\prime} \times X \rightarrow \mathbb{R}$ is a bicontraction. Using the universal property $\left(^{*}\right)$ 
we obtain that $\tilde{e v}=i d:\left(X^{\prime},|\cdot|_{\mu \vee \eta}\right) \rightarrow\left(X^{\prime}, \mathcal{M}_{X^{\prime}}\right)$ is a contraction. Therefore $\xi \leq|\cdot|_{\eta \vee \mu}$. Now it follows from (3.4) and (3.5) that $|\cdot|_{\eta}=$ $|\cdot|_{\eta \vee \mu}$. From the Hahn-Banach theorem it follows $\eta=\eta \vee \mu$, that is, $\mu \leq \eta$.

We have therefore shown that $X$ is a seminormed space.

Conversely let $(X, \eta)$ be a seminormed space. Fix $Y \in|\mathbf{l c A p V e c}|$. For all $\mu \in \mathcal{M}_{Y}$ we have that the map $\mu_{\eta}: Y^{X} \rightarrow \mathbb{R}^{+}: a \mapsto \sup _{\eta(x) \leq 1} \mu(a(x))$ is well defined and a seminorm, where $Y^{X}$ is the set of all linear continuous maps from $X$ to $Y$. Let $\mathcal{M}_{Y X}$ be the Minkowski system generated by the base $\left\{\mu_{\eta} \mid \mu \in \mathcal{M}_{Y}\right\}$. Then $e v: Y^{X} \times X \rightarrow Y$ is a bicontraction; let $\overline{e v}$ be the linear lift. Take $f: Z \otimes X \rightarrow Y$ a linear contraction. For all $z \in Z$ the map $\hat{f}(z): X \rightarrow Y: x \mapsto f(z \otimes x)$ is linear and continuous. Moreover $\hat{f}: Z \rightarrow Y^{X}$ is a linear contraction: let $\xi \in \mathcal{M}_{Y^{X}}$, pick $\mu \in \mathcal{M}_{Y}$ such that $\xi \leq \mu_{\eta}$. There exists $\nu \in \mathcal{M}_{Z}$ such that $\mu(f(z \otimes x)) \leq \nu(z) \eta(x)$. Thus we have $\xi(\hat{f}(z)) \leq \mu_{\eta}(\hat{f}(z)) \leq \nu(z)$. It is straightforward to verify that $\hat{f}$ is unique with the property that $f=\overline{e v} \circ(\hat{f} \otimes i d)$.

The projective tensor product does not define a monoidal closed structure on lcApVec. However since the projective tensor product of seminormed spaces is again a seminormed space, we obtain the following well-known fact as a simple corollary:

Corollary 3.10. The projective tensor product restricted to sNorm defines a symmetrical monoidal closed structure. If $(X, \eta)$ and $(Y, \mu)$ are seminormed spaces, the seminorm on $Y^{X}$ is given by the well known seminorm

$$
\mu_{\eta}=\sup _{\eta(x) \leq 1} \mu(f(x))
$$

where $f$ is a continuous linear function from $X$ to $Y$.

In particular, when $Y=(\mathbb{R},|\cdot|)$ in the above, we obtain the strong dual of $X$.

\section{REFERENCES}

[1] Adámek J., Herrlich H. and Strecker G., Abstract and concrete categories, J. Wiley and Sons, 1990.

[2] Borceux F. Handbook of Categorical Algebra, Vol. I,II,III, Encyclopedia of Math. and its Appl., Cambridge University Press, 1996.

[3] Bourbaki N. Eléments de mathématique, livre V, Espaces Vectoriels Topologiques, Hermann, Paris 1964.

[4] Grothendieck A. Produits tensoriels topologiques et espaces nucléaires, Mem. Amer. Math Soc. 16,1955.

[5] Lowen R. and Sioen M. Approximations in Functional Analysis, Result. Math. 37 (2000) $345-372$.

[6] Lowen R. and Verwulgen S. Approach Vector Spaces, submitted.

[7] Lowen R. and Windels B. Approach groups, Rocky Mountain J. of Math 30(3) (2000) 1057-1074.

[8] Lowen R. Approach spaces A common Supercategory of TOP and Met, Math. Nachr. 141 (1989) 183-226. 
[9] Lowen R. Approach Spaces: The Missing Link in the Topology-Uniformity-Metric Triad, Oxford Mathematical Monographs, Oxford University Press, 1997.

[10] Mac Lane S. Categories for the working mathematician, Springer 1997.

[11] Pumplün D. and Röhrl H. Banach Spaces and Totally Convex Spaces I, Communications in algebra, 12(8) (1984) 953-1019.

[12] Pumplün D. and Röhrl H. Banach Spaces and Totally Convex Spaces II, Communications in algebra 13(5) (1985) 1047-1113.

[13] Pumplun D. Eilenberg-Moore algebras revisited, Seminarberichte, FB Mathematik und Informatic, Fernuniversität 29 (1988) 57-144.

[14] Rudin W. Functional analysis, Intern. Series in Pure and Appl. Math., McGraw-hill, 1991.

[15] Ryan R.A. Introduction to Tensor Product of Banach spaces, Springer Monographs in Math., Springer Verlag (London), 2002.

[16] Schaefer H.H. Topological vector spaces, Graduate Texts in Mathematics, Springer, 1999.

[17] Sydow W. On Hom-functors and tensor products of topological vector spaces, Lecture notes in Math. 962 (1982) 292-301.

[18] Schatten R. A theory of cross spaces, Annals of math. studies 26, Princeton university press, 1950.

[19] Von Neumann J. On infinite direct products, Compositio Math. 6 (1938) 1-77.

Received November 2001

REvised SEPTEMBER 2002

M. SiOEN

Departement Wiskunde, Vrije Universiteit Brussel, Pleinlaan 2, 1050 Brussel, Belgium

E-mail address: msioen@vub.ac.be

\section{S. VERWULGEN}

Departement Wiskunde-Informatica, Universiteit Antwerpen, Middelheimlaan 1, 2020 Antwerpen, Belgium

E-mail address: stijn.verwulgen@ua.ac.be 